\title{
Changes in Photosynthetic Characteristics and Antioxidative Protection in Male and Female Ginkgo during Natural Senescence
}

\author{
Dawei Shi \\ College of Life Sciences, Nanjing Normal University, Nanjing 210046, P.R. China; and the College \\ of Forest Resources and Environment, Nanjing Forestry University, Nanjing 210037, P.R. China
}

Xiaodong Wei

College of Life Sciences, Nanjing Normal University, Nanjing 210046, P.R. China; and the Institute of Food and Crops, Jiangsu Academy of Agricultural Sciences, Nanjing 210014, P.R. China

Guoxiang Chen ${ }^{1}$

College of Life Sciences, Nanjing Normal University, Wenyuan Road 1, Nanjing 210046, P.R. China

Yanli Xu

College of Life Sciences, Nanjing Normal University, Nanjing 210046, P.R. China

AdDitional Index words. Ginkgo biloba, photosynthesis, antioxidative responses, chloroplast ultrastructure, sexual differences

\begin{abstract}
Ginkgo (Ginkgo biloba), a dioecious tree species, is widely distributed throughout the world, yet little is known about sex-related responses to autumnal senescence in ginkgo. The aim of this study was to investigate changes in photosynthetic activities, concentration of oxidative stress parameters [malondialdehyde (MDA) and $\mathrm{H}_{2} \mathrm{O}_{2}$ ] and antioxidant systems, and ultrastructure of chloroplasts in the naturally senescing leaves of two ginkgo sexes and to examine whether progression of senescence is sex-specific in ginkgo. Photosynthesis in ginkgo leaves of both sexes was not limited by stomatal factors, but rather non-stomatal factors such as decreased photosynthetic pigments and photochemical activities that became more important during autumnal senescence. The responses of antioxidative enzymes were different from those of antioxidants to leaf senescence. Correlation analysis revealed that autumnal leaf senescence was significantly correlated to antioxidative enzymes changes but not to antioxidants such as ascorbate (ASA) and glutathione (GSH). Guaiacol peroxidase (POD) became more important in senescing leaves and played a major protective role, especially at the late stage of senescence. The shape of chloroplasts of both sexes changed from oblong to round, and there was an increase in the number and size of osmiophilic granules during senescence; swollen thylakoid membranes in the stroma and grana with a significant increase in MDA content were also observed. During autumnal senescence, female ginkgo plants showed smaller decreases in net photosynthetic rates, photosynthetic pigments, photochemical activities, superoxide dismutase, ascorbate peroxidase and catalase activities, higher POD activity, ASA and GSH contents, and smaller increases in $\mathrm{H}_{2} \mathrm{O}_{2}$ and MDA contents than did males. In addition, female plants had a later senescence of chloroplasts, a smaller accumulation of osmiophilic granules, and a slower rate of membrane damage. These results show that female ginkgo exhibit slower leaf senescence, which may be related to increased reproductive costs.
\end{abstract}

Leaf senescence is characterized by programmed degradation of cellular constituents such as proteins, nucleic acids, and lipids, together with organelles and structures of leaf cell, resulting in a significant photosynthetic decline. Photosynthesis is a vital physiological factor for the biomass and grain yield. However, initiation of grain filling coincides with the onset of leaf senescence, offsetting the grain yield. So the onset and rate of senescence are important factors for determining grain yield

\footnotetext{
Received for publication 8 Feb. 2012. Accepted for publication 19 July 2012. This research was financially supported by the Natural Science Foundation of Jiangsu Higher Education Institutions (Grant No. 11KJA180001) and a project founded by the Priority Academic Program Development of Jiangsu High Education Institutions (PAPD).

We thank Fred Chow of Australian National University of Canberra, Canberra, Australia, for his help in improving the English of the article and Sergi MuuneBosch of Barcelona University, Barcelona, Spain, for his constructive criticism. We also gratefully acknowledge both three anonymous reviewers and the ASHS editors for helpful suggestions.

${ }^{1}$ Corresponding author. E-mail: chengxnjnu@163.com.
}

(Subhan and Murthy, 2001; Zhang et al., 2006). In addition, the different responses of Populus cathayana to photoperiod transitions may be related to its different leaf senescence speed under changing environments (Zhao et al., 2009). Therefore, early or rapid senescence can lead to productivity losses or affect the growth of plants.

Ginkgo is a long-lived, deciduous gymnosperm species that is resistant to pests and pollutants. The extracts of ginkgo leaves contain flavonoid glycosides and terpenoids and have been used pharmaceutically (Cao, 2002). Ginkgo leaves are usually harvested in the fall, because the contents of these pharmaceutical constituents are highest in fall and then decline with senescence in ginkgo leaves (Chen et al., 1997). In addition, this harvest date coincides with the onset of autumnal leaf senescence (Cheng et al., 2009). Therefore, modification of cultural practices may help to delay senescence and result in economic benefits to cultivating this plant.

Chloroplasts are the sites of photosynthesis, and a decline in photosynthesis corresponds with ultrastructural alterations in 
the chloroplast as a leaf progresses toward senescence (Quirino et al., 2000). One of the major events in leaf senescence is the increase of reactive oxygen species (ROS) levels, which can result in a decline in antioxidant protection, including both enzymatic and non-enzymatic factors (Zimmermann and Zentgraf, 2005). Superoxide dismutases (SOD), catalases (CAT), and various peroxidases such as guaiacol peroxidase and ascorbate peroxidase (APX) are the primary antioxidant enzymes. In conjunction with these enzymes, antioxidant compounds such as ascorbate and glutathione also play important roles in maintaining the integrity of photosynthetic membranes under oxidative stress (Kuk et al., 2003).

Many morphological, physiological, and ecological differences between males and females have been observed in a number of dioecious species in relation to environmental stresses (Rozas et al., 2009; Stehlik et al., 2008). Because of the differences in reproductive resource requirements, females are more common in high-resource microsites, whereas males are more tolerant to environmental stresses (Zhang et al., 2010b, 2011). Although it has been reported that male and female ginkgo have similar responses to light (Jin et al., 2008), little is known about the sex-specific progress to senescence.

It has been well documented that in ginkgo and other dioecious plants, males senesce earlier and faster than females because male plants are more susceptible to infection (Molisch, 1929). Changes in the antioxidant status of ginkgo leaves during senescence have been reported (Kukavica and VeljovicJovanovic, 2004). However, this study was primarily focused on a single sex. In this study, we used natural conditions of leaf senescence to investigate whether progress of senescence is sexspecific in ginkgo leaves (Zhang et al., 2010a). The aim of the present work is to compare a series of changes in photosynthetic and antioxidative activity and chloroplast ultrastructure in male and female plants during autumnal senescence to assess which sex senesces earlier or more rapidly. We believe that this comparison between sexes could be used to develop strategies to increase the growth and productivity of ginkgo.

\section{Materials and Methods}

Plant material. The experiments were performed using the leaves of 30-year-old ginkgo trees (female and male) standing in pairs on the campus of Nanjing Normal University in Nanjing, China (lat. $32^{\circ} 03^{\prime} \mathrm{N}$, long. $118^{\circ} 45^{\prime} \mathrm{E}$ ). Nanjing is located in the monsoon climate area of the north subtropical zone with four distinctive seasons (Fan and Chen, 1997). Weather conditions are given in Figure 1. From 1 Sept. (16 d before the first sampling) to 13 Nov. (the last sampling date), the mean maximum and minimum temperature was (mean $\pm \mathrm{SE}$ ) $24.9 \pm 0.4{ }^{\circ} \mathrm{C}$ and $16.2 \pm 0.5^{\circ} \mathrm{C}$, respectively. The mean $( \pm \mathrm{SE})$ daily precipitation and relative humidity was $1.4 \pm 0.5 \mathrm{~mm}$ and $75.8 \% \pm 1.2 \%$, respectively.

The ginkgo leaves emerge in early April, then expand from April to June, and senesce in late September in male and female trees. Three trees of each sex with similar age $(\approx 30$ years old $)$ were sampled from 16 Sept. 2006 (close to the time of leaf yellowing, Day 259) through 13 Nov. 2006 (close to the time of leaf drop, Day 317 ) on sunny mornings (0730 to $1130 \mathrm{HR}$ ) every 6 to $16 \mathrm{~d}$, depending on the weather. The leaves used for analyses were fully expanded and from lateral branches of the outer part of the crowns with the same exposure to light.

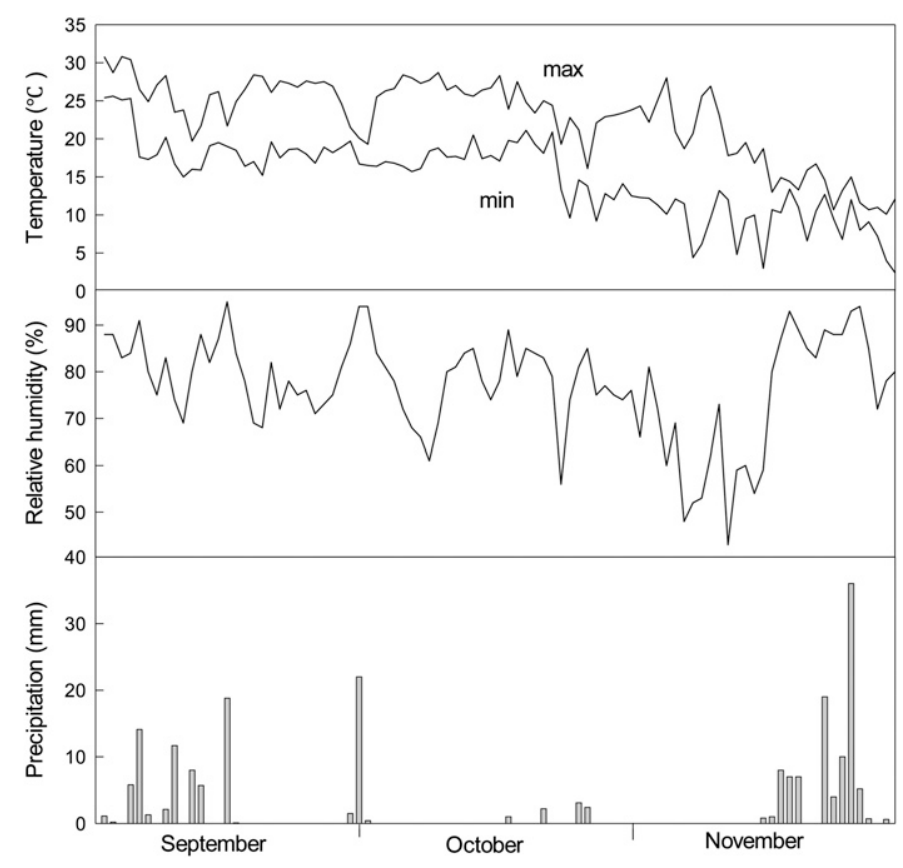

Fig. 1. Precipitation, relative humidity, and maximum/minimum temperatures during the study period (September to November).

Pigment determinations. Leaves $(n=5)$ of each tree were detached at each sampling point, immediately frozen in liquid nitrogen, and stored at $-80^{\circ} \mathrm{C}$ until further analysis. Each leaf sample was extracted in ice-cold $80 \%(\mathrm{v} / \mathrm{v})$ acetone. The extract was centrifuged at $3000 \mathrm{~g}_{\mathrm{n}}$ for $5 \mathrm{~min}$. The absorbance of the supernatant was measured with a spectrophotometer (GENESYS 10UV; Thermo Fisher Scientific, Waltham, MA) at 470, 645, and $663 \mathrm{~nm}$. The levels of chlorophyll (Chl) and carotenoids were calculated as described by Lichtenthaler and Wellburn (1983).

Рнотosynthetic activity measurements. The net photosynthetic rates $\left(\mathrm{P}_{\mathrm{n}}\right)$, stomatal conductance $\left(g_{\mathrm{S}}\right)$, and intercellular $\mathrm{CO}_{2}$ concentration $(\mathrm{Ci})$ were measured in the field on sunexposed leaves $(n=10$ leaves per tree) using a portable photosynthesis system (CIRAS-2; PP Systems, Amesbury, MA) in the morning between 0730 and 1130 HR. To avoid potential photoinhibition from high light stress, the measurements were conducted under saturating photosynthetic photon flux densities $\left(1000 \mu \mathrm{mol} \cdot \mathrm{m}^{-2} \cdot \mathrm{s}^{-1}\right)$ from a light-emitting diode (red and white) light source. The leaves in the cuvette were exposed to ambient $\mathrm{CO}_{2}$ concentration $\left(\approx 360 \mu \mathrm{mol} \cdot \mathrm{mol}^{-1}\right)$, relative humidity, and temperature.

To further elucidate changes in the primary photochemical reactions of photosynthesis during senescence, photosynthetic electron transport and photophosphorylation were investigated using thylakoids isolated from ginkgo leaves. Approximately $2 \mathrm{~g}$ of leaves, without petioles $(\mathrm{n}=5)$, were taken from each tree and stored at $4{ }^{\circ} \mathrm{C}$ for $30 \mathrm{~min}$ at each sampling time until further analysis. The membranes were isolated as described by Zhang et al. (2006). Photophosphorylation activity was measured with a luminescent photometer (FG-300; Shanghai Institute of Plant Physiology, Shanghai, China) as described by Ketcham et al. (1984). The $\mathrm{Ca}^{2+}$-ATPase activity was measured according to Vallejos et al. (1983). The adenosine-5' -triphosphate (ATP) content was measured using the bioluminescence method described by Zhu et al. (2001). The electron transport 
activities [photosystem I (PSI), photosystem II (PSII), and whole-chain activities] were measured polarographically with a Clark-type liquid-phase electrode (Chlorolab-2; Hansatech, Norfolk, U.K.) fitted with a circulating water jacket at $25 \pm 0.5^{\circ} \mathrm{C}$.

$\mathrm{H}_{2} \mathrm{O}_{2}$ Determination. For the $\mathrm{H}_{2} \mathrm{O}_{2}$ determination, $1 \mathrm{~g}$ of leaves $(\mathrm{n}=5)$ was harvested from each tree and homogenized in $5 \%(\mathrm{v} / \mathrm{v}) \mathrm{HClO}_{4}$. The suspension was centrifuged at $14,000 g_{\mathrm{n}}$ for $10 \mathrm{~min}$ at $4{ }^{\circ} \mathrm{C}$. The supernatant was neutralized with $5 \mathrm{M} \mathrm{K}_{2} \mathrm{CO}_{3}$ to reach $\mathrm{pH} 4$, then centrifuged at $14,000 \mathrm{~g}_{\mathrm{n}}$ for $2 \mathrm{~min}$ at $4{ }^{\circ} \mathrm{C}$, and immediately used to evaluate the $\mathrm{H}_{2} \mathrm{O}_{2}$ content according to Veljovic-Jovanovic et al. (2002).

LIPID PEROXIDATION AND ANTIOXIDANT ENZYMES ANALYSIS. For this analysis, $1 \mathrm{~g}$ of leaves $(\mathrm{n}=5)$ was harvested from each tree, pulverized in liquid nitrogen using a mortar and pestle, and extracted with $0.05 \mathrm{M}$ phosphate buffer ( $\mathrm{pH}$ 7.8) containing $5 \mathrm{~mm}$ EDTA. The suspension was centrifuged at $10,000 g_{\mathrm{n}}$ for $20 \mathrm{~min}$ at $4{ }^{\circ} \mathrm{C}$. The supernatant was used to assay for SOD, POD, CAT, and malondialdehyde. For the APX assay, the extraction buffer also contained $2 \mathrm{~mm}$ ascorbate.

The level of MDA production was assayed to estimate lipid peroxidation according to the method described by Zhao and $\mathrm{Li}$ (1999). The MDA in the supernatant was considered to be a thiobarbituric acid-reactive substance. The absorbance was recorded at 532, 600, and $450 \mathrm{~nm}$. The SOD activity was determined using the procedure described by Giannopolitis and Ries (1977). The activity was assayed by the capacity of SOD to inhibit the reduction of nitro-blue tetrazolium (NBT) by xanthine oxidase-generated $\mathrm{O}_{2}^{-}$, producing an increase in the absorbance at $560 \mathrm{~nm}$ of $\approx 0.02$ units per minute at $25^{\circ} \mathrm{C}$ in the absence of enzyme. One unit of SOD activity was defined as the amount of enzyme required to inhibit $50 \%$ of the initial rate of NBT reduction. The CAT activity was assayed according to the method described by Mishra et al. (1993). The enzyme activity was determined by monitoring the change in optical density (OD) at $240 \mathrm{~nm}$ and was defined as the decrease of $0.1 \triangle \mathrm{OD}$ per minute. The APX activity was determined by monitoring the decline in absorbance at $290 \mathrm{~nm}$ as ascorbate is oxidized, according to the method described by Nakano and Asada (1981). One unit of APX activity was defined as the decrease of $0.1 \triangle \mathrm{OD}$ per minute. The POD activity was assayed according to the method described by Kochba et al. (1977). The change of OD was recorded at $470 \mathrm{~nm}$ and the enzyme activity was defined as the increase of $0.1 \triangle \mathrm{OD}$ per minute.

Ascorbate AND GLUTATHIONE Determinations. The ginkgo leaves $(0.5 \mathrm{~g}, \mathrm{n}=5$ per tree $)$ were ground with a mortar and pestle in trichloroacetic acid at $4{ }^{\circ} \mathrm{C}$. The homogenate was centrifuged at $13,000 g_{\mathrm{n}}$ for $15 \mathrm{~min}$, and the supernatant was collected for ASA and GSH analyses. The ASA and GSH pools were assayed according to Tanaka et al. (1985) and Ellman (1959), respectively. The concentrations of ASA and GSH were calculated from a standard curve prepared from known concentrations of ASA and GSH.

Transmission Electron microscopy. To avoid differential structure in different parts of leaves, the midsection of the leaves without the midrib was used and cut into small pieces $(2 \times 20 \mathrm{~mm})$. The pieces were fixed at room temperature in $4 \%$ (v/v) glutaraldehyde dissolved in $0.3 \mathrm{M}$ sodium phosphate buffer ( $\mathrm{pH} 7.5$ ) for $2 \mathrm{~h}$. After washing in buffer, the samples were postfixed with $1 \%$ osmium tetroxide in the same buffer. After $2 \mathrm{~h}$, the samples were dehydrated in graded acetone solutions and embedded in Epon 812 resin. The resin was polymerized at $60{ }^{\circ} \mathrm{C}$. Ultrathin sections $(70 \mathrm{~nm})$ were cut with a diamond knife on a microtome (LKB-V ultramicrotome; LKB, Bromma, Sweden). The sections were stained with uranyl acetate, followed by lead citrate, and observed using a transmission electron microscope (TEM 600A-2; Hitachi, Tokyo, Japan). To attain sufficient accuracy, two sections per leaf piece, three pieces per leaf, and three leaves per tree were observed at different stages of senescence.

Statistical ANAlyses. Analysis of variance and correlation were based on the general linear model procedure. Statistical differences between sexes and between different dates with respect to photosynthetic pigments and activities, $\mathrm{H}_{2} \mathrm{O}_{2}$ content, lipid peroxidation, and antioxidant systems were analyzed by performing an analysis of variance using SPSS (Version 16.0; IBM Corp., Armonk, NY). Differences were considered significant at the $P<0.05$ level.

\section{Results}

The photosynthetic pigments and photosynthetic rates were determined in sun-exposed leaves of ginkgo plants over $58 \mathrm{~d}$ as biomarkers of autumnal leaf senescence (Garcia-Plazaola et al., 2003).

Both chlorophyll and carotenoid levels were highest by Day 266 and subsequently decreased in both sexes (Fig. 2). After Day 282, a decrease in the $\mathrm{Chl}$ a/b ratio was also observed, indicating that $\mathrm{Chl}$ a content was more affected by leaf aging. The decrease in the carotenoid content was less than that of Chl content. Males showed greater decreases in Chl, carotenoids, and $\mathrm{Chl} \mathrm{a} / \mathrm{b}$ ratio than females under natural conditions. Females possessed significantly higher Chl than males after Day $282(P=0.001)$. There were no sex-specific differences in the carotenoid content and $\mathrm{Chl} \mathrm{a} / \mathrm{b}$ ratio during most of leaf growth. However, males had lower values at the late stage of leaf senescence.

The $\mathrm{P}_{\mathrm{n}}$ and $g_{\mathrm{S}}$ were negatively affected by aging in both sexes. As shown in Figure 3, marked decreases in these two parameters were observed after Day 266. However, the $\mathrm{Ci}$ remained stable throughout the process of senescence. The change in Chl content correlated with the decreased $\mathrm{P}_{\mathrm{n}}$ during leaf senescence. Females possessed higher $\mathrm{P}_{\mathrm{n}}, g_{\mathrm{S}}$, and $\mathrm{Ci}$ than males during leaf senescence; however, males showed greater decreases in $\mathrm{P}_{\mathrm{n}}$.

Taken together, the data on photosynthetic pigment contents and photosynthetic rates indicated that the ginkgo leaves at Day 266 (September) were mature, and the leaves after this time showed progressive stages of senescence.

As shown in Figure 4, the decrease in the PSII electron transport activity was larger than that of the PSI and the whole electron chain, indicating that PSII was more sensitive during the process of senescence of ginkgo leaves. Although males had higher electron transport values during the early stage of leaf senescence, males showed larger decreases in electron transport than females, especially at the late stage of senescence.

As shown in Figure 5, photophosphorylation, $\mathrm{Ca}^{2+}$-ATPase activity and ATP content demonstrated a similar trend during leaf yellowing, reaching the highest levels at Day 266 and then decreasing significantly. During senescence of ginkgo leaves (especially during the middle and late stages), the higher ATP content in combination with the higher $\mathrm{Ca}^{2+}$-ATPase and photophosphorylation activities in females suggests that the turnover of ATP was higher in females than in males. Before leaf senescence, males displayed higher $\mathrm{Ca}^{2+}$-ATPase activity 


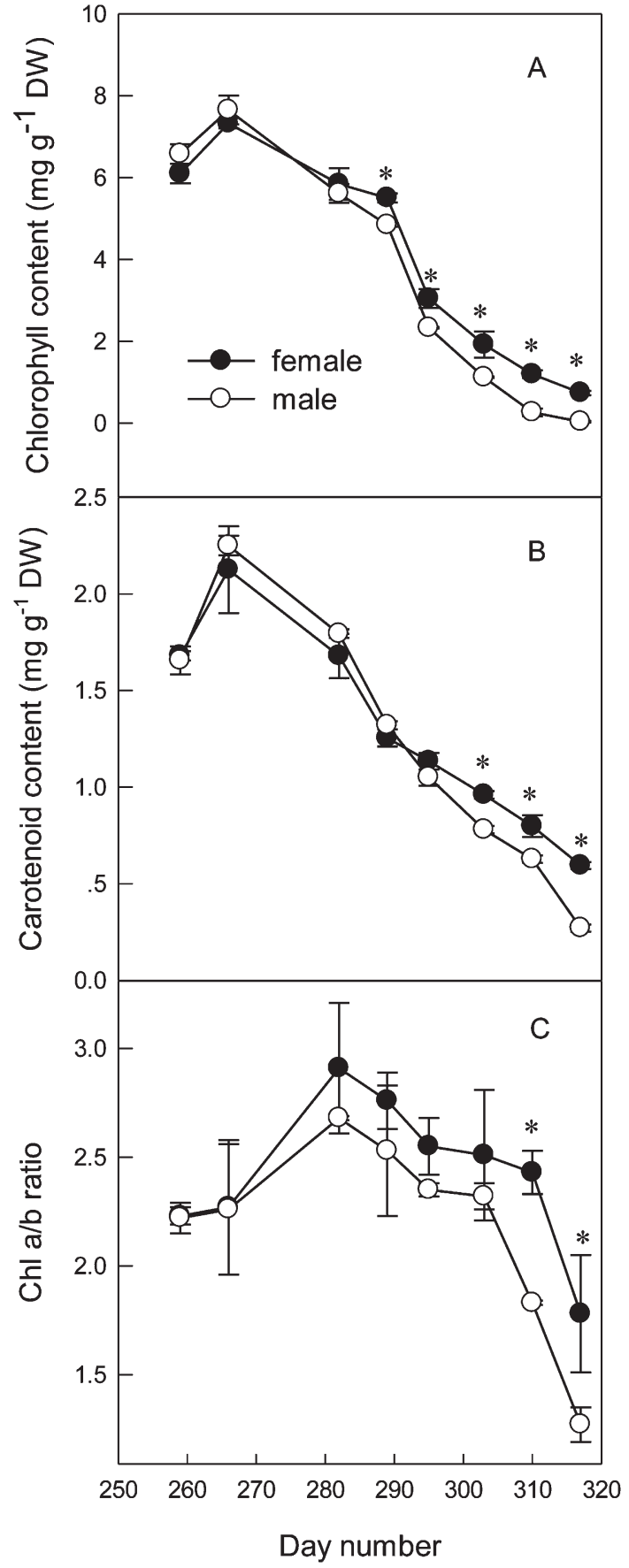

Fig. 2. Changes in chlorophyll (A) and carotenoid (B) contents and chlorophyll $\mathrm{a}$ to $\mathrm{b}$ ratio $[\mathrm{Chl} \mathrm{a} / \mathrm{b}(\mathbf{C})]$ in female (filled symbols) and male (open symbols) ginkgo leaves during autumnal senescence. Values represent the average of 15 replicates $\pm \mathrm{SE}$. Single asterisks indicate statistically significant differences $(P \leq$ 0.05 ) between female and male ginkgo during the same period; Day $1=1$ Jan.

and ATP content, which might be the reason for their higher $\mathrm{P}_{\mathrm{n}}$ at this stage (Miginiac-Maslow and Lancelin, 2002).

The hydrogen peroxide content and the level of lipid peroxidation, measured in terms of MDA content, increased with leaf age in both sexes, reaching the highest values in the late stage of senescence at Day 317 (Fig. 6). Moreover, the sexspecific differences were significant with males showing higher $\mathrm{H}_{2} \mathrm{O}_{2}$ and MDA contents than females after Day $282\left(\mathrm{H}_{2} \mathrm{O}_{2}, P=\right.$ 0.014 ; MDA, $P=0.007$ ). Significant sex-specific differences

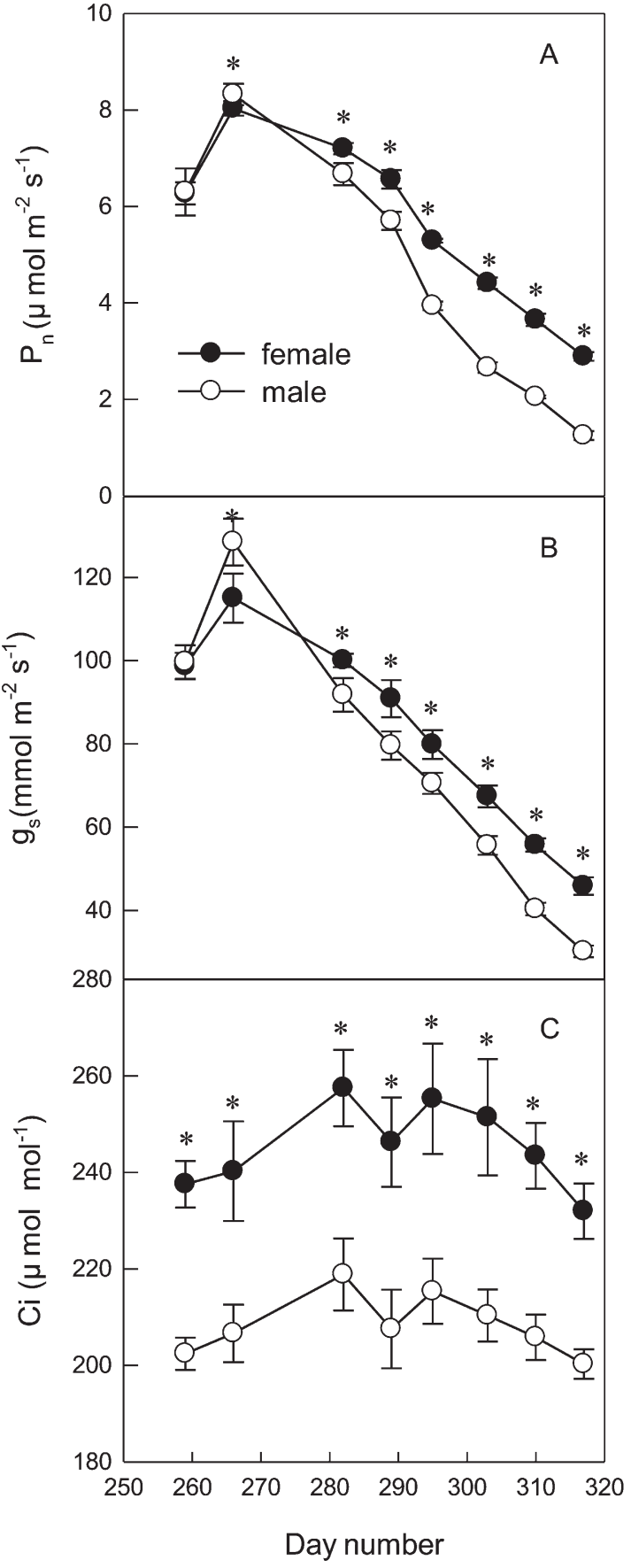

Fig. 3. Changes in net photosynthetic rate $\left[\mathrm{P}_{\mathrm{n}}(\mathbf{A})\right]$, stomatal conductance $\left[g_{\mathrm{S}}(\mathbf{B})\right]$, and internal $\mathrm{CO}_{2}$ concentration $[\mathrm{Ci}(\mathbf{C})]$ in female (filled symbols) and male (open symbols) ginkgo leaves during autumnal senescence. Values represent the average of 30 replicates \pm SE. Single asterisks indicate statistically significant differences $(P \leq 0.05)$ between female and male ginkgo during the same period; Day $1=1$ Jan.

were also observed in ASA and GSH contents during leaf yellowing (ASA, $P=0.001$; GSH, $P=0.001$ ). However, ASA and GSH contents remained at a relatively stable level in both sexes until the late stage of leaf senescence. Females showed higher ASA and GSH contents during leaf senescence.

The changes in the activities of primary antioxidative enzymes (SOD, APX, CAT, and POD) are shown in Figure 7. 


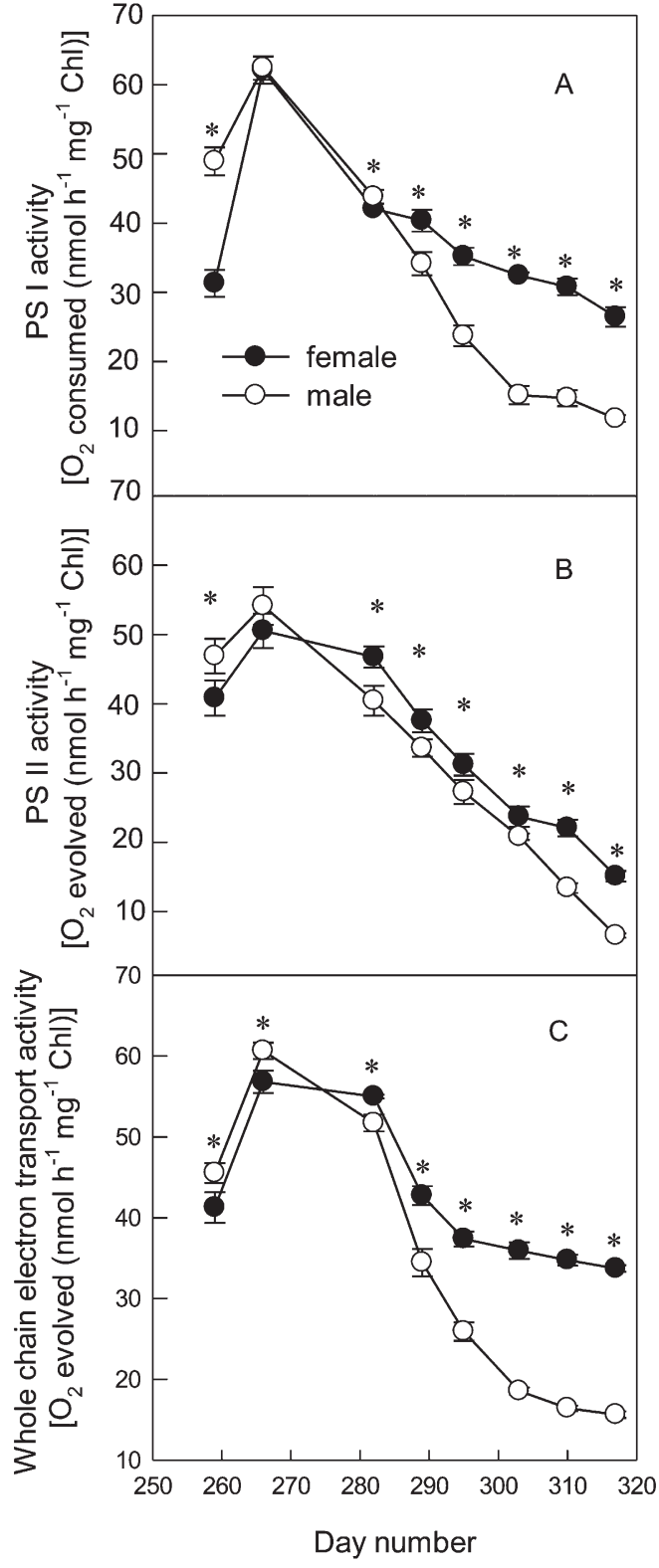

Fig. 4. Changes in photosystem I [PSI (A)], photosystem II [PSII (B)], and whole-chain electron transport activities (C) in female (filled symbols) and male (open symbols) ginkgo leaves during autumnal senescence. Values represent the average of 15 replicates \pm SE. Single asterisks indicate statistically significant differences $(P \leq 0.05)$ between female and male ginkgo during the same period; Day $1=1 \mathrm{Jan} . \mathrm{Chl}=$ chlorophyll.

The activities of SOD, CAT, and APX increased, reaching their highest values at Day 266 and then decreasing significantly, which is probably the result of increased oxidative stress during the process of leaf senescence. Males exhibited greater decreases in the activities of these three enzymes than females. In contrast, POD activity increased significantly in the senescent leaves of both sexes, but was much higher in females than in males $(P=0.001)$.

Correlations showed that $g_{\mathrm{S}}$ was positively correlated to senescence parameters $\left(\mathrm{P}_{\mathrm{n}}, \mathrm{Chl}\right.$, and carotenoid contents), whereas $\mathrm{Ci}$ had no significant correlations with these parameters in both sexes (Tables 1 and 2). There were significant positive correlations of PSI, PSII, and whole-chain electron

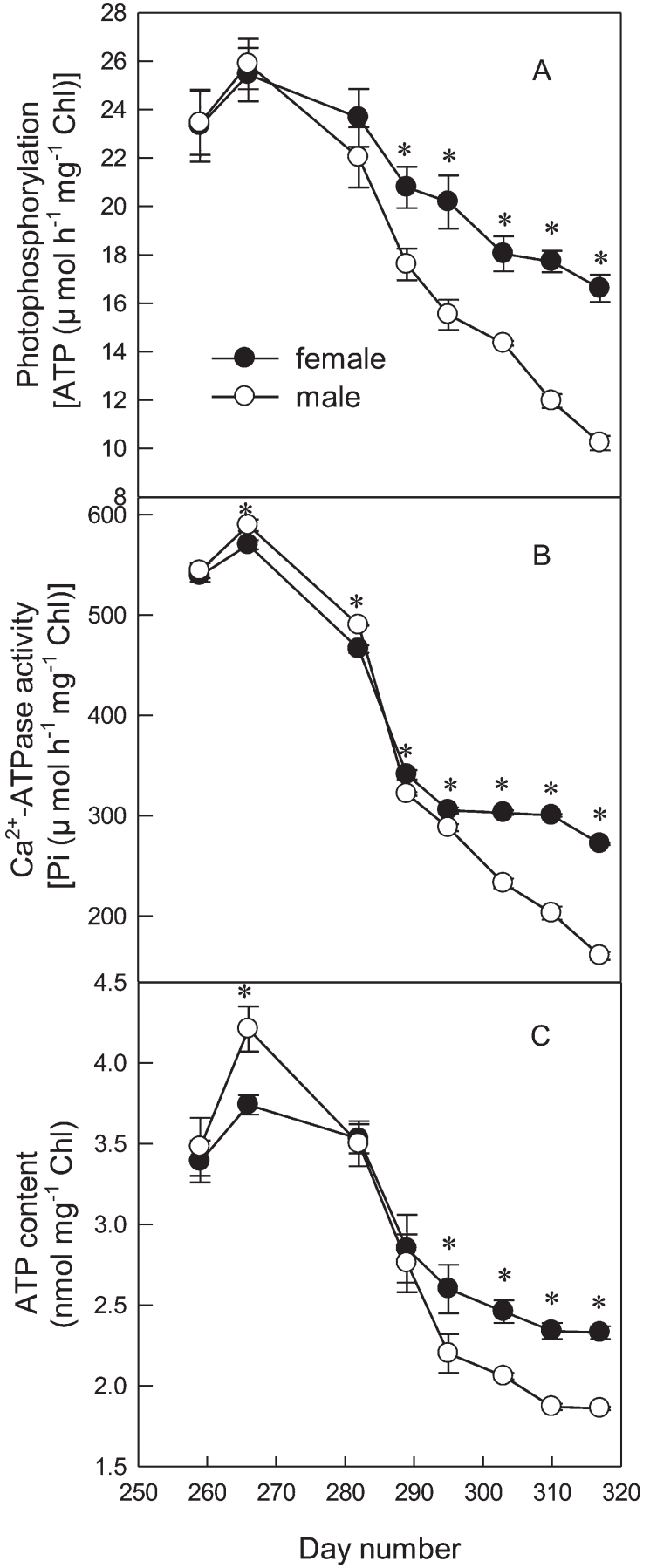

Fig. 5. Changes in photophosphorylation (A), $\mathrm{Ca}^{2+}$-ATPase activity (B), and adenosine triphosphate (ATP) content (C) in female (filled symbols) and male (open symbols) ginkgo leaves during autumnal senescence. Values represent the average of 15 replicates \pm SE. Single asterisks indicate statistically significant differences $(P \leq 0.05)$ between female and male ginkgo during the same period; Day $1=1 \mathrm{Jan}$. $\mathrm{Chl}=$ chlorophyll.

transport activities, photophosphorylation, $\mathrm{Ca}^{2+}$-ATPase activity, ATP content, SOD, CAT, and APX activities with all senescence parameters for both sexes. POD activity, $\mathrm{H}_{2} \mathrm{O}_{2}$, and MDA contents also correlated with these parameters, but negatively. No significant correlations were detected between ASA, GSH contents, and senescence parameters. 

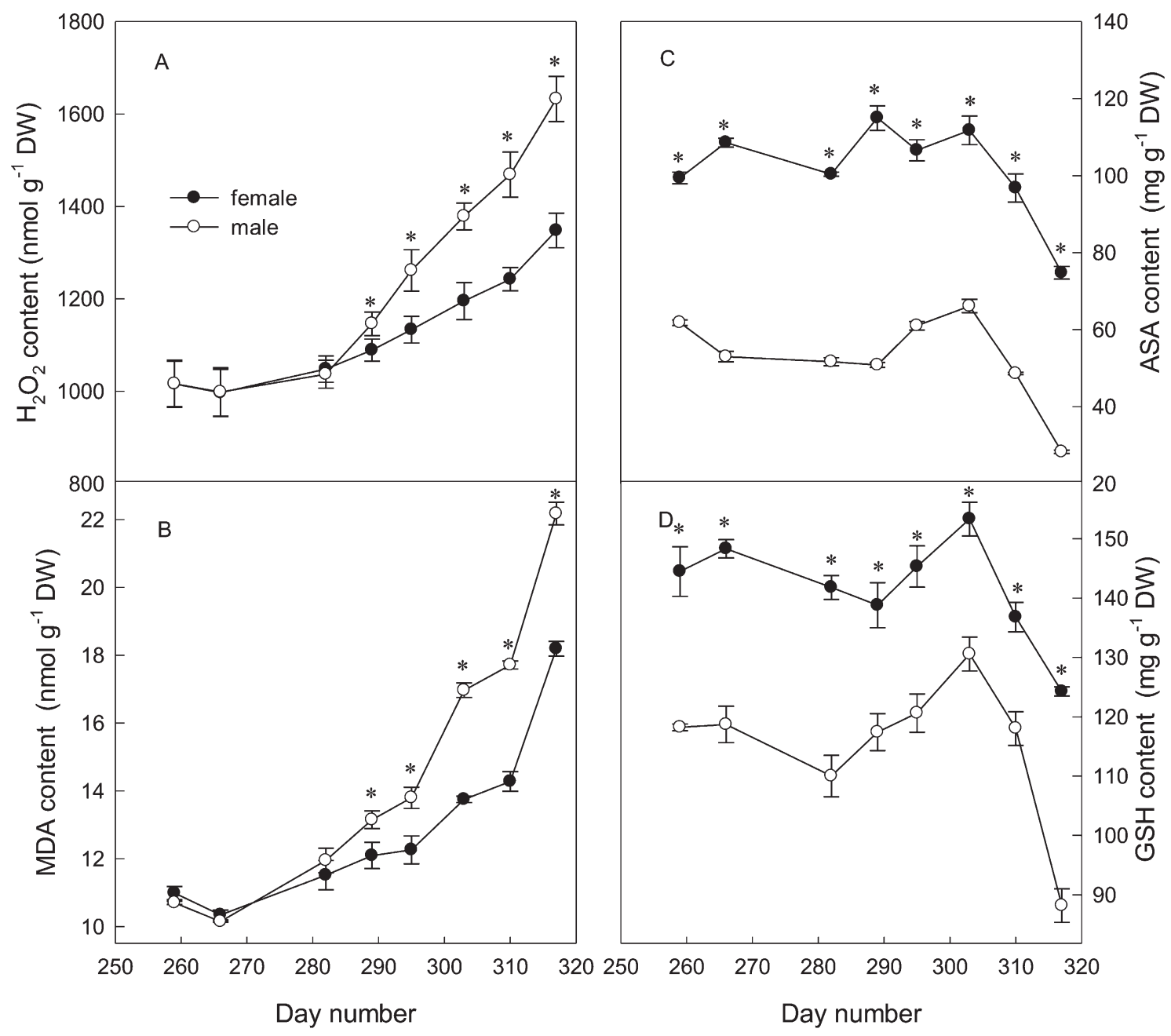

Fig. 6. Changes in hydrogen peroxide $\left[\mathrm{H}_{2} \mathrm{O}_{2}\right.$ (A)], malondialdehyde [MDA (B)], ascorbic acid [ASA (C)], and glutathione [GSH (D)] contents in female (filled symbols) and male (open symbols) ginkgo leaves during autumnal senescence. Values represent the average of 15 replicates \pm SE. Single asterisks indicate statistically significant differences $(P \leq 0.05)$ between female and male ginkgo during the same period; Day $1=1$ Jan.

As shown in Figures 8 and 9, at the early stage of leaf senescence, the chloroplasts from both sexes exhibited a normal ultrastructural organization; most were lens-like oblong-shaped with a typical arrangement of grana and stroma thylakoids; the appearance of osmiophilic granules was observed at this stage. Chloroplasts from females and males changed in their shapes from Day 295 and Day 289, respectively. They were usually lens-shaped but they were more rounded than chloroplasts at the early stage of leaf senescence. The number and size of the osmiophilic granules changed slightly in females, whereas more and large osmiophilic granules were observed in males. In addition, the thylakoids of grana and stroma were swollen and reduced. At Day 310, close to the time of leaf drop, the ultrastructure of the chloroplasts from both sexes changed significantly. The peripheral double membrane of the chloroplasts and the internal system of grana and stroma became disorganized in both males and females; the stratiform structure of the chloroplasts became inconspicuous, and the parallel membrane was disrupted. The osmiophilic granules became more and larger in the chloroplast of females. Moreover, the thylakoids became swollen and the intrathylakoid space was increased. In comparison, the osmiophilic granules were developed further in males, and the number and the size increased significantly. The thylakoids in the chloroplasts from males were severely damaged, and the membrane structure was almost lost.

\section{Discussion}

A decline in photosynthesis has been reported in many plants during leaf senescence (Cabello et al., 2006; Zhang et al., 2007). In this study, marked decreases in $P_{n}$ were observed in both sexes after Day 266 in ginkgo leaves, and females exhibited higher values than males. An increase of photorespiration was observed with a decrease of $g_{\mathrm{S}}$ as a result of stomatal closure in ginkgo leaves exposed to high light, which inhibited photosynthesis (Meng et al., 1999). In the present study, the decrease of $g_{\mathrm{S}}$ was attributed to stomatal closure in senescent ginkgo leaves. However, the Ci remained constant in both sexes during senescence, which indicated that the decrease in $\mathrm{P}_{\mathrm{n}}$ was not the result of a decrease in the available $\mathrm{CO}_{2}$ as a result of decreased $g_{\mathrm{S}}$ but possibly the result of non-stomatal factors such as a decline in Chl content, decreased biochemical activities, and damage to the photosynthetic system (Whitehead 


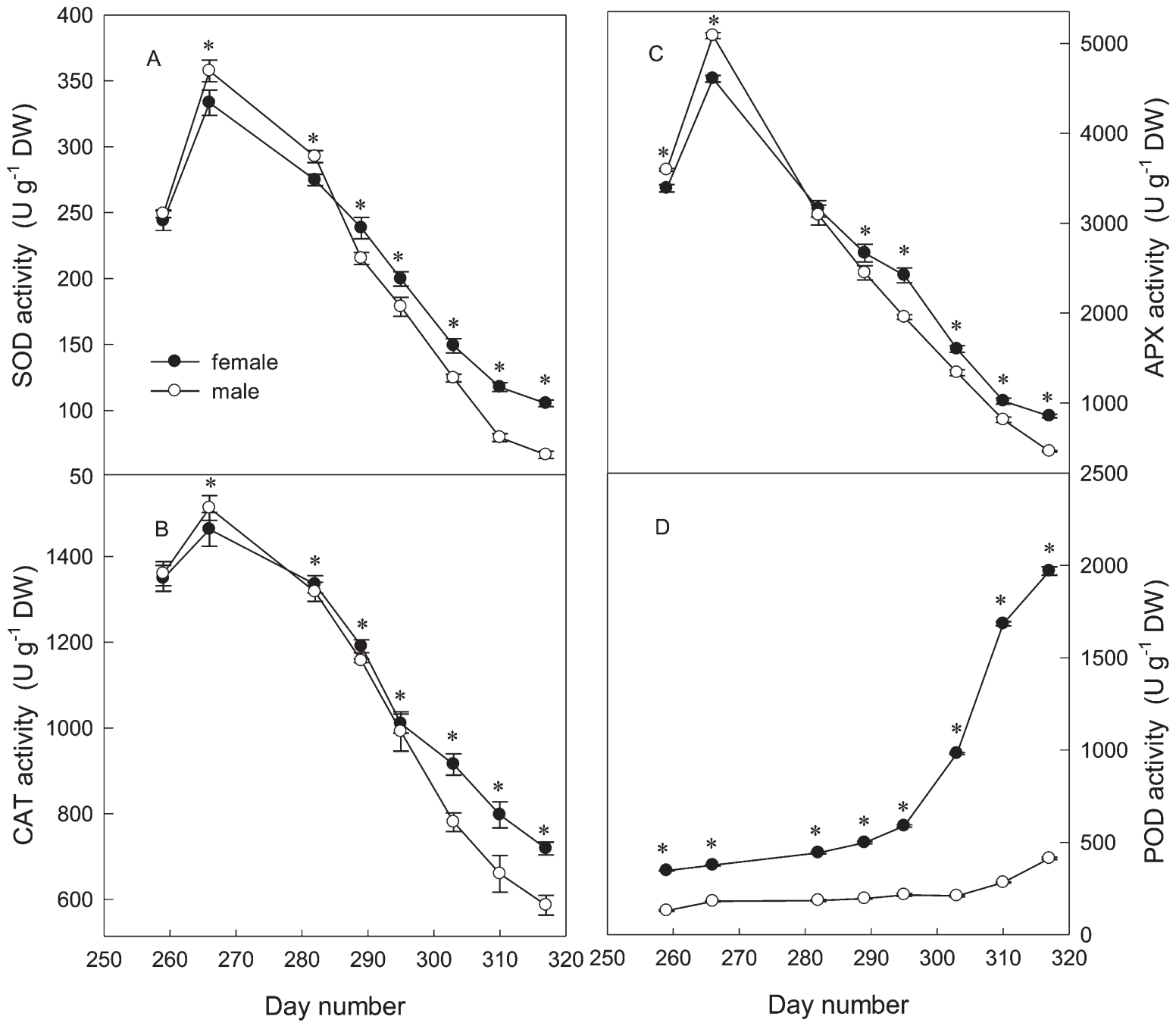

Fig. 7. Changes in superoxide dismutase [SOD (A)], catalase [CAT (B)], ascorbate peroxidase [APX (C)], and peroxidase [POD (D)] activities in female (filled symbols) and male (open symbols) ginkgo leaves during autumnal senescence. Values represent the average of 15 replicates \pm SE. Single asterisks indicate statistically significant differences $(P \leq 0.05)$ between female and male ginkgo during the same period; Day $1=1$ Jan.

et al., 2011). This result is consistent with the report by He et al. (2007). Actually, the Chl content was significantly decreased in both sexes, and females exhibited higher Chl than males during leaf senescence. Leaf yellowing results from chlorophyll degradation and is widely used as a phenotype marker of plant senescence together with a series of other biochemical and physiological changes (Ougham et al., 2008). Higher Chl content indicated that the process of leaf senescence was delayed in female ginkgo plants. The $\mathrm{Chl} \mathrm{a} / \mathrm{b}$ ratio might play an important role in controlling leaf senescence, and the decrease in this ratio may be in favor for the plants before late senescence (Zhang et al., 2006). The $\mathrm{Chl} \mathrm{a} / \mathrm{b}$ ratio was greater in females during late senescence after Day 303, showing that the females had a slower rate of leaf senescence. During ginkgo leaf senescence, the Chls were more susceptible to degradation than carotenoids. Carotenoids have been reported to protect chloroplasts against blue light during autumnal senescence (Garcia-Plazaola et al., 2003). The decrease of carotenoid content was greater in males, which indicated that the senescence of chloroplasts might be faster in males than in females.
Leaf senescence generally induces an alteration in the structure and function of the chloroplasts concomitant with a decrease in photosynthetic activity. Previous studies have shown that leaf senescence also induces a decrease in the photochemical activities of both PSII and PSI, and in most cases, PSII is more responsive to senescence than PSI (Zhu et al., 2001). Similar results were also observed in this study. Females showed smaller decreases in the activities of PSII, PSI, and whole-chain electron transport during autumnal senescence. However, the photosystem activities were higher in males at the early stage of leaf senescence. The change pattern was similar to that of male and female cottonwood (Letts et al., 2008) or spinach (Sklensky and Davies, 2011) plants. This may be the result of higher leaf maturity in males than in females, because males showed increased Chl and $P_{n}$ (Liu et al., 2011). Photophosphorylation activity is the capacity of chloroplasts to produce ATP under light conditions, indicating the light energy conversion ability of chloroplasts. Similarly, after Day 289 (middle and late senescence), the photophosphorylation, $\mathrm{Ca}^{2+}$-ATPase activity, and ATP content were higher in females than in males. These results suggest that higher photochemical 


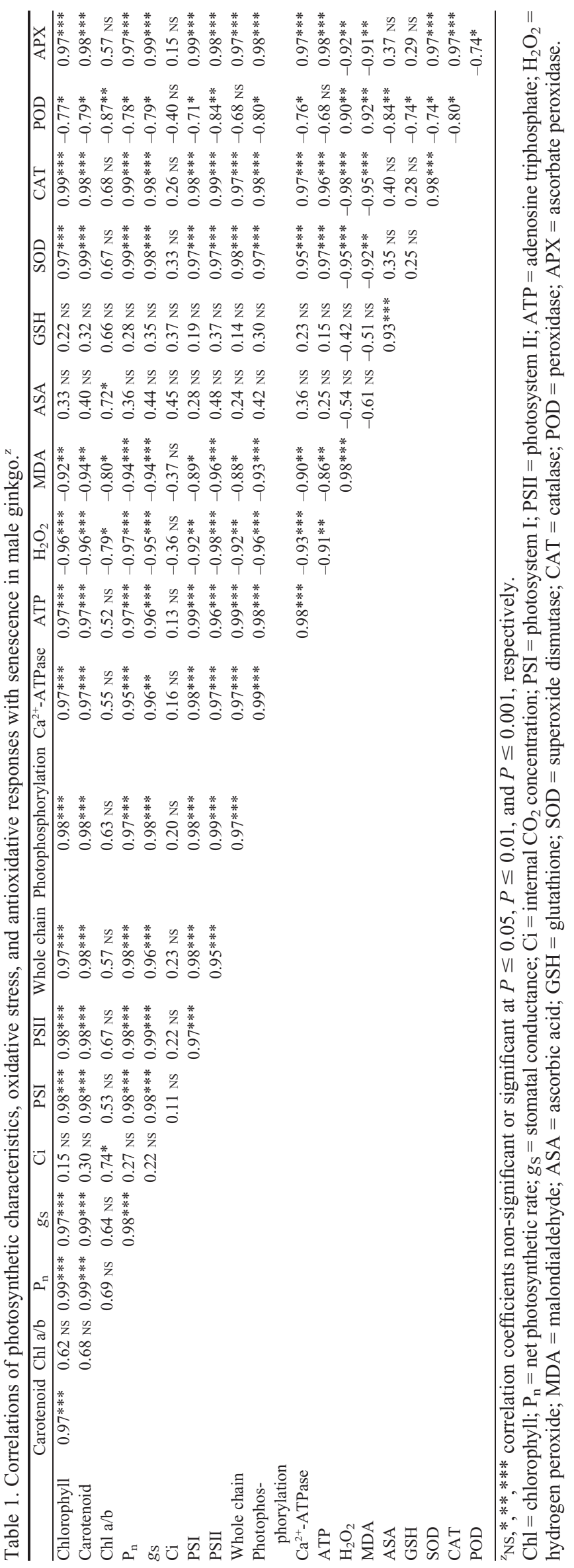

activities may be responsible for the higher photosynthetic rate in females.

Plant aging increases oxidative stress and ROS levels (Zimmermann and Zentgraf, 2005). Chloroplasts are the primary source of ROS and are the main target of age-associated oxidative stress in plants (Asada, 2006). In the thylakoids, $\mathrm{Chl}$ is complexed with carotenoids, which prevents the occurrence of activated oxygen by either quenching triplet states of Chl or scavenging singlet oxygen and thereby dissipating excessive energy (Matile, 2001). Therefore, the lower levels of Chl and carotenoids resulted in the accumulation of more ROS in males, which might be supported by an increase of hydrogen peroxide. MDA is a common product of membrane lipid peroxidation. Lipid peroxidation is an integral feature of membrane deterioration leading to cell death and is correlated with oxidative stress during leaf senescence (Zimmermann and Zentgraf, 2005). In this study, MDA content significantly increased in both sexes and males exhibited a higher value than females during the process of senescence. Higher hydrogen peroxide and MDA suggest that males suffered more oxidative stress and greater damage to the cellular membrane or chloroplast structure. This result is consistent with the report on P. cathayana by Zhao et al. (2009).

The antioxidant protection generally decreased during senescence, and $\mathrm{H}_{2} \mathrm{O}_{2}$ regulation may play a critical role in the onset and dynamics of senescence. The SODs are metalloenzymes that catalyze the dismutation of a superoxide radical to molecular oxygen and $\mathrm{H}_{2} \mathrm{O}_{2}$. The decrease in SOD activity that we observed during leaf senescence suggested that a decrease in $\mathrm{H}_{2} \mathrm{O}_{2}$ levels might occur; however, this was not the case. Indeed, there was an increase of $\mathrm{H}_{2} \mathrm{O}_{2}$ with ginkgo leaf aging. This increase might be the result of the decreased scavenging activity of $\mathrm{H}_{2} \mathrm{O}_{2}$ in both sexes. Three important antioxidative enzymes (APX, CAT, and POD) are responsible for $\mathrm{H}_{2} \mathrm{O}_{2}$ detoxification in cell compartments, and there are some reports about the increase of POD activity and the decrease of APX and CAT activities during leaf senescence (Hodges and Forney, 2001; Kar and Mishra, 1976; VeljovicJovanovic et al., 2006). The ASA-GSH cycle is also an important antioxidant protection system against $\mathrm{H}_{2} \mathrm{O}_{2}$, and its occurrence has been considered to be associated with leaf senescence (Foyer and Shigeoka, 2011; Palma et al., 2006). In ginkgo leaves, the ASA and GSH levels stayed relatively constant until Day 303, when a significant increase in $\mathrm{H}_{2} \mathrm{O}_{2}$ content was detected. This and the decreased APX and CAT activities, with increased POD activity, indicated that in senescent plants, ASA could not perhaps be used to scavenge $\mathrm{H}_{2} \mathrm{O}_{2}$ by the ASA-GSH cycle and declines in the APX and CAT activities may contribute to the increased $\mathrm{H}_{2} \mathrm{O}_{2}$ during the early stage of senescence. In the late stage of senescence, the decreases in ASA and GSH were accompanied by continuous POD activity increase and progressive inactivation of APX and CAT. These results suggested that POD could still participate in removing $\mathrm{H}_{2} \mathrm{O}_{2}$ when the photosynthetic apparatus has been severely damaged, which were consistent with those reported by Kukavica and Veljovic-Jovanovic (2004). In addition, sexspecific responses in antioxidative defenses were observed in ginkgo during the process of senescence. Females showed smaller decreases in SOD, APX, and CAT activities, higher POD activity and antioxidant contents, and slower increases in $\mathrm{H}_{2} \mathrm{O}_{2}$ and lipid peroxidation levels than males. These results suggest that females have a better protection mechanism than 


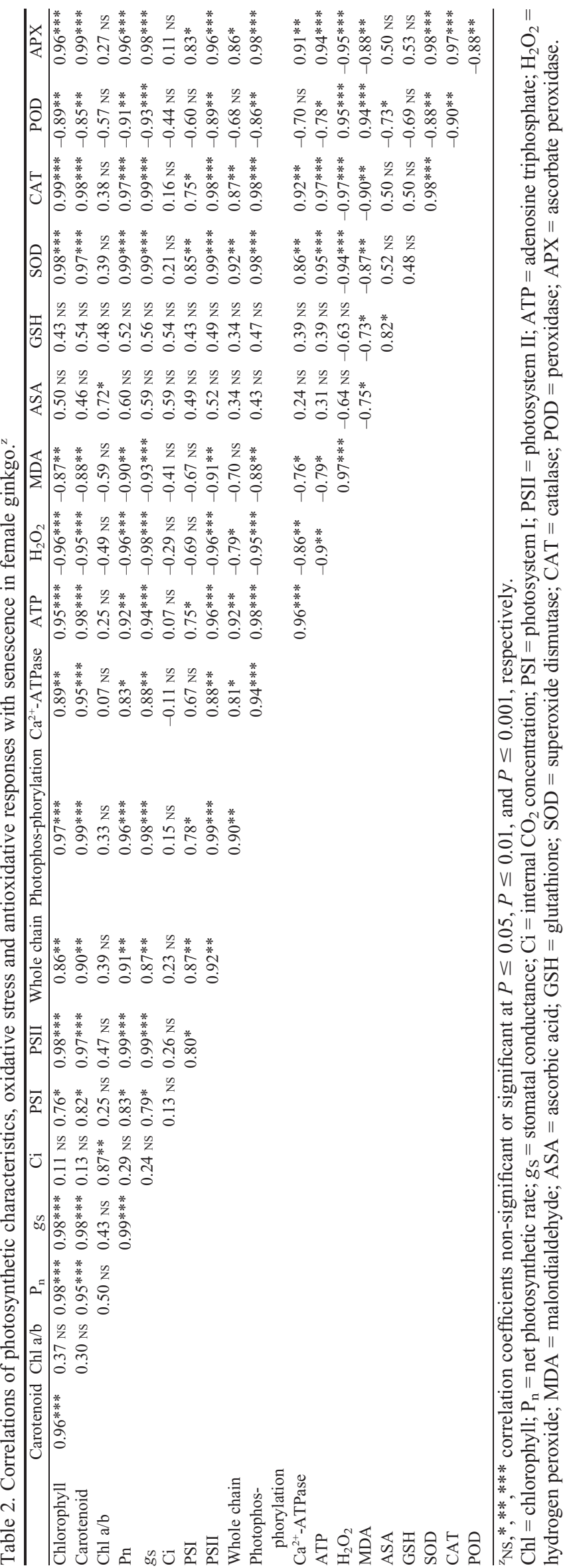

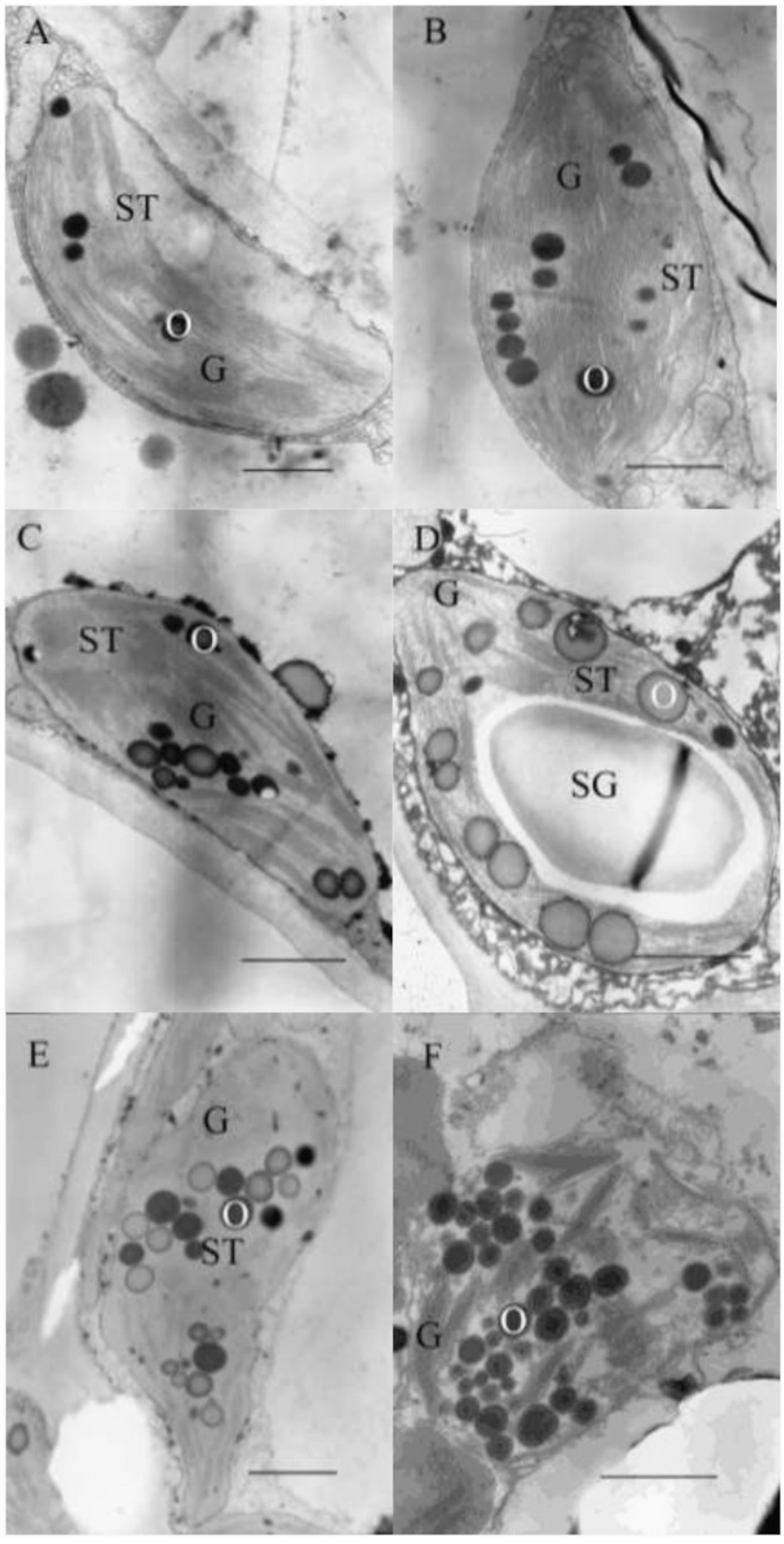

Fig. 8. Changes in the ultrastructure of chloroplasts from female ginkgo leaves during autumnal senescence: (A) Day 266, (B) Day 282, (C) Day 289, (D) Day 295, (E) Day 303, (F) Day 310. G = granum thylakoid; ST = stroma thylakoid; $\mathrm{O}=$ osmiophilic granule; $\mathrm{SG}=$ starch grain. $\mathrm{Bars}=1 \mu \mathrm{m}$; Day $1=$ 1 Jan.

males for ROS detoxification to delay leaf senescence to meet their greater resource demands.

Data from correlation analysis demonstrated that photochemical activity decreases, antioxidative enzymes inactivation or activation, $\mathrm{H}_{2} \mathrm{O}_{2}$, and lipid peroxidation accumulation were significantly correlated natural senescence; however, the correlation with stomatal closure and antioxidants were not significant. Moreover, photochemical activities were positively correlated with photosynthesis, whereas stomatal closure was not. POD activity was positively correlated and another three 


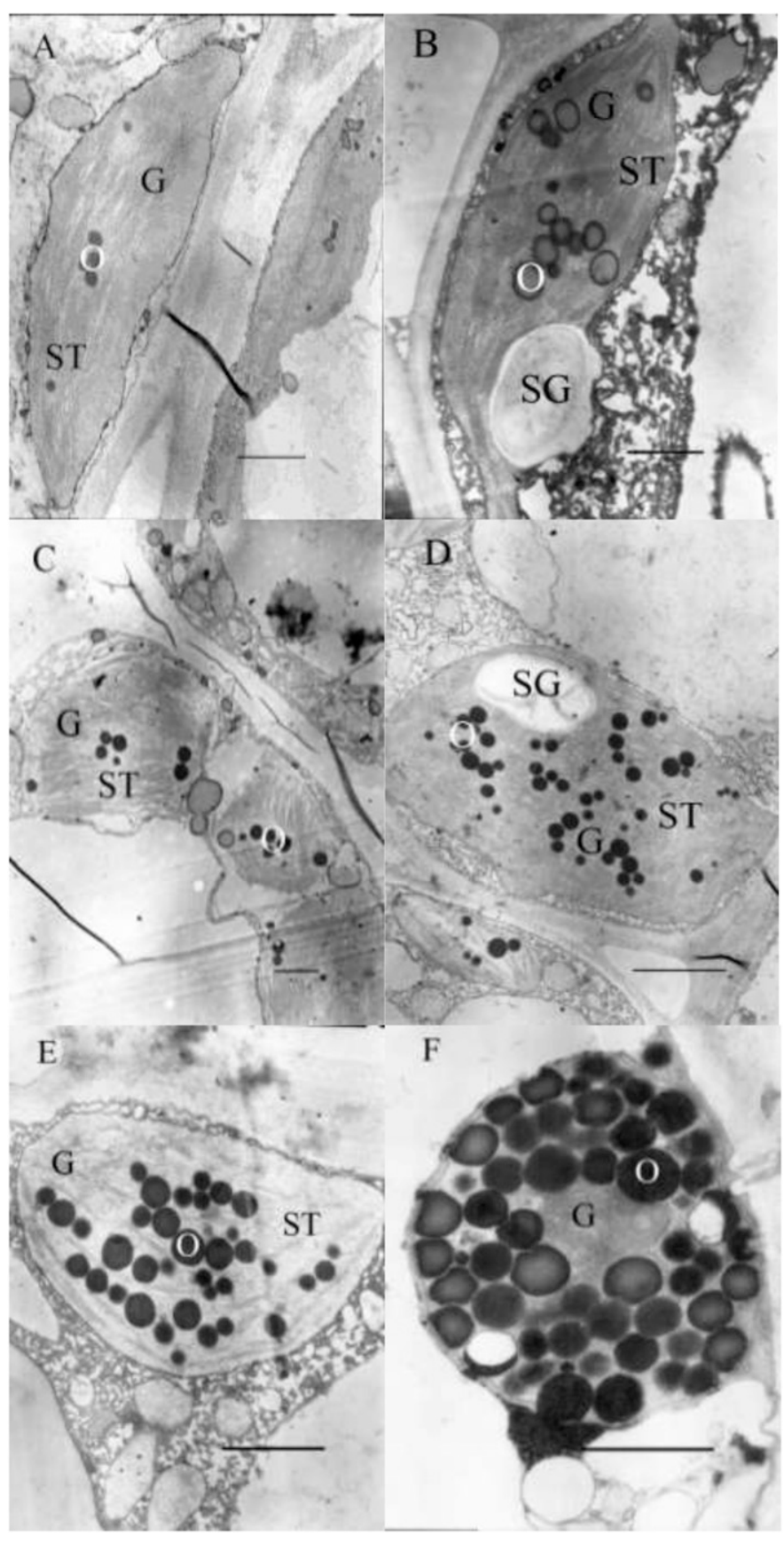

Fig. 9. Changes in the ultrastructure of chloroplasts from male ginkgo leaves during autumnal senescence: (A) Day 266, (B) Day 282, (C) Day 289, (D) Day 295, (E) Day 303, (F) Day 310. G = granum thylakoid; ST = stroma thylakoid; $\mathrm{O}=$ osmiophilic granule; $\mathrm{SG}=$ starch grain. Bars $=1 \mu \mathrm{m}$; Day $1=1 \mathrm{Jan}$.

antioxidative enzymes were negatively correlated with oxidative stress, whereas the correlations with antioxidants were not significant. Thus, approaches that can increase photochemical activities or activate antioxidative enzymes may be useful to delay senescence of ginkgo leaves.

The ultrastructural changes were apparent in ginkgo leaves during senescence. Chloroplasts are one of the first organelles targeted for breakdown during leaf senescence (Quirino et al., 2000). In this study, we found that the chloroplasts senesced similarly, but the dynamics of senescence were different in females and males. After Day 266 (the beginning of senescence), the shape of chloroplasts changed from oblong to round in both sexes (earlier in males at Day 289 than in females at Day 295). The osmiophilic granules increased remarkably in size and number (larger in males than in females), which is a typical feature of chloroplast aging (Spundova et al., 2003). The membrane system of grana and stroma thylakoids became reduced and swollen, which resulted in the disorganization of the parallel pattern of the lamellae and a considerable change in the orientation of the grana. During the last stage of senescence, the membrane structure was almost lost filled with osmiophilic granules in the chloroplasts of males, but not in females. The senescence of chloroplasts in aspen (Populus tremula) was different from that of female ginkgo, but similar to that of male ginkgo, which may be attributed to earlier fall leaf senescence (Keskitalo et al., 2005). An increase in the number and size of osmiophilic granules resulted from a gradual thylakoid membrane degradation (Papadakis et al., 2004). These results revealed that females exhibited a slower rate of chloroplast breakdown under natural conditions than males. Moreover, the delayed Chl breakdown and maintenance of photosynthesis were accompanied by a less rapid breakdown of chloroplasts in female ginkgo.

In conclusion, we observed more rapid leaf senescence of males was related to sex-specific differences in photosynthesis and antioxidative protection. In both sexes, the decrease in photosynthesis was caused by non-stomatal limitation of ginkgo leaves. Males showed a greater decrease in photosynthetic pigments, reduced photochemical activities, a lower antioxidative capacity to defend against oxidative stress and a more rapid degradation of chloroplast membrane structure than did females.

Females of woody dioecious usually expend proportionally more of their resources on reproduction and less on maintenance and growth when compared with males (Obeso, 2002). However, high reproductive costs or sink strength (e.g., fruit or seeds) can be accompanied with increased leaf photosynthesis and antioxidative activities, which delays leaf senescence (Kaschuk et al., 2010; Sklensky and Davies, 2011; Zhao et al., 2009). In some female plants such as Silene latifolia, fruit may be contributed to the differences on photosynthetic rates throughout the flowering period among those individuals (Laporte and Delph, 1996). Therefore, it is hypothesized that female ginkgo may respond to greater cost of reproduction through a higher capacity to delay the decline in photosynthesis and better protection against oxidative stress, which could offset the respiratory costs and maintain its reproductive organs, whereas male ginkgo may not (Case and Ashman, 2005).

\section{Literature Cited}

Asada, K. 2006. Production and scavenging of reactive oxygen species in chloroplasts and their functions. Plant Physiol. 141:391-396.

Cabello, P., E. Aguera, and P. de la Haba. 2006. Metabolic changes during natural ageing in sunflower (Helianthus annuus) leaves: Expression and activity of glutamine synthetase isoforms are regulated differently during senescence. Physiol. Plant. 128:175185

Cao, F.L. 2002. Chinese ginkgo. Jiangsu Science and Technology Press, Nanjing, China.

Case, A.L. and T.L. Ashman. 2005. Sex-specific physiology and its implications for the cost of reproduction, p. 129-158. In: Reekie, E.G. and F.A. Bazzaz (eds.). Reproductive allocation in plants. Academic Press, New York, NY.

Chen, X.S., W.C. Zhang, and X.X. Deng. 1997. Seasonal changes of the contents of flavonoids and ginkgolides in the leaves of Ginkgo 
biloba and their changes of different stages of development for the tree. J. Fruit Sci. 14:226-229.

Cheng, S.Y., F. Xu, and Y. Wang. 2009. Advances in the study of flavonoids in Ginkgo biloba leaves. J. Medicinal Plants Res. 3:12481252.

Ellman, G.L. 1959. Tissue sulfhydryl groups. Arch. Biochem. Biophys. 82:70-77.

Fan, J.S. and K.X. Chen. 1997. Tendency features of precipitation variation in Nanjing in this century. Acta Meteorologica Sinica 17:237-245.

Foyer, C.H. and S. Shigeoka. 2011. Understanding oxidative stress and antioxidant functions to enhance photosynthesis. Plant Physiol. 155:93-100.

Garcia-Plazaola, J.I., A. Hemandez, and J.M. Becerril. 2003. Antioxidant and pigment composition during autumnal leaf senescence in woody deciduous species differing in their ecological traits. Plant Biol. 5:557-566

Giannopolitis, C.N. and S.K. Ries. 1977. Superoxide dismutases: I. Occurrence in higher plants. Plant Physiol. 59:309-314.

He, X.Y., S.L. Fu, W. Chen, T.H. Zhao, S. Xu, and Z. Tuba. 2007. Changes in effects of ozone exposure on growth, photosynthesis, and respiration of Ginkgo biloba in Shenyang urban area. Photosynthetica 45:555-561.

Hodges, D.M. and C.F. Forney. 2001. Antioxidant responses in harvested leaves of two cultivars of spinach differing in senescence rates. J. Amer. Soc. Hort. Sci. 126:611-617.

Jin, J., H. Jiang, S.Q. Yu, and G.M. Zhou. 2008. Sex-linked photosynthetic physiologic research and the evolutionary ecological analysis in living fossil plant, Ginkgo biloba L. Acta Ecol. Sin. 28:1128-1136.

Kar, M. and D. Mishra. 1976. Catalase, peroxidase, and polyphenoloxidase activities during rice leaf senescence. Plant Physiol. 57:315-319.

Kaschuk, G., M. Hungria, P.A. Leffelaar, K.E. Giller, and T.W. Kuyper. 2010. Differences in photosynthetic behaviour and leaf senescence of soybean [Glycine max (L.) Merrill] dependent on $\mathrm{N}_{2}$ fixation or nitrate supply. Plant Biol. 12:60-69.

Keskitalo, J., G. Bergquist, P. Gardestrom, and S. Jansson. 2005. A cellular timetable of autumn senescence. Plant Physiol. 139:16351648.

Ketcham, S.R., J.W. Davenport, K. Warncke, and R.E. MaCarty. 1984. Role of the $\gamma$ subunit of chloroplast coupling factor 1 in the lightdependent activation of phosphorylation and ATPase activity by dithiothreitol. J. Biol. Chem. 259:7286-7293.

Kochba, J., S. Lavee, and P. Spiegel-Roy. 1977. Differences in peroxidase activity and isoenzymes in embryogenic and nonembryogenic 'Shamouti' orange ovular callus lines. Plant Cell Physiol. 18:463-467.

Kuk, Y.I., J.S. Shin, N.R. Burgos, T.E. Hwang, O. Han, B.H. Cho, S. Jung, and J.O. Guh. 2003. Antioxidative enzymes offer protection from chilling damage in rice plants. Crop Sci. 43:2109-2117.

Kukavica, B. and S. Veljovic-Jovanovic. 2004. Senescence-related changes in the antioxidant status of ginkgo and birch leaves during autumn yellowing. Physiol. Plant. 122:321-327.

Laporte, M.M. and L.F. Delph. 1996. Sex-specific physiology and source-sink relations in the dioecious plant Silene latifolia. Oecologia 106:63-72.

Letts, M.G., C.A. Phelan, D.R.E. Johnson, and S.B. Rood. 2008. Seasonal photosynthetic gas exchange and leaf reflectance characteristics of male and female cottonwoods in a riparian woodland. Tree Physiol. 28:1037-1048.

Lichtenthaler, H.K. and A.R. Wellburn. 1983. Determinations of total carotenoids and chlorophylls $a$ and $b$ of leaf extracts in different solvents. Biochem. Soc. Trans. 11:591-592.

Liu, Y., Z.C. Zhong, X.X. Wang, J. Xie, and W.Y. Yang. 2011. Photosynthesis and transpiration characteristics of female and male Trichosanthes kirilowii Maxim individuals. Chinese J. Appl. Ecol. 22:644-650.
Matile, P. 2001. Senescence and cell death in plant development: Chloroplast senescence and its regulation, p. 277-296. In: Aro, E.M. and B. Andersson (eds.). Regulation of photosynthesis. Kluwer, Dordrecht, The Netherlands.

Meng, Q.W., E. Weis, Q. Zou, and S.J. Zhao. 1999. Photoinhibition and photoprotection in Ginkgo biloba leaves: Influence of temperature, $\mathrm{CO}_{2}$ and $\mathrm{O}_{2}$. Acta Bot. Sin. 41:398-404.

Miginiac-Maslow, M. and J.M. Lancelin. 2002. Intrasteric inhibition in redox signalling: Light activation of NADP-malate dehydrogenase. Photosynth. Res. 72:1-12.

Mishra, N.P., R.K. Mishra, and G.S. Singhal. 1993. Changes in the activities of anti-oxidant enzymes during exposure of intact wheat leaves to strong visible light at different temperatures in the presence of protein synthesis inhibitors. Plant Physiol. 102:903-910.

Molisch, H. 1929. Die Lebensdauer der Pflanze. Fischer Verlag, Jena, Germany.

Nakano, Y. and K. Asada. 1981. Hydrogen peroxide is scavenged by ascorbate-specific peroxidase in spinach chloroplasts. Plant Cell Physiol. 22:867-880.

Obeso, J.R. 2002. The costs of reproduction in plants. New Phytol. 155:321-348.

Ougham, H., S. Hortensteiner, I. Armstead, I. Donnison, I. King, H. Thomas, and L. Mur. 2008. The control of chlorophyll catabolism and the status of yellowing as a biomarker of leaf senescence. Plant Biol. 10(suppl 1):4-14.

Palma, J.M., A. Jimenez, L.M. Sandalio, F.J. Corpas, M. Lundqvist, M. Gomez, F. Sevilla, and L.A. del Rio. 2006. Antioxidative enzymes from chloroplasts, mitochondria, and peroxisomes during leaf senescence of nodulated pea plants. J. Expt. Bot. 57:17471758.

Papadakis, I.E., K.N. Dimassi, A.M. Bosabalidis, I.N. Therios, A. Patakas, and A. Giannakoula. 2004. Boron toxicity in 'Clementine' mandarin plants grafted on two rootstocks. Plant Sci. 166:539-547.

Quirino, B.F., Y.S. Noh, E. Himelblau, and R.M. Amasino. 2000. Molecular aspects of leaf senescence. Trends Plant Sci. 5:278-282.

Rozas, V., L. DeSoto, and J.M. Olano. 2009. Sex-specific, agedependent sensitivity of tree-ring growth to climate in the dioecious tree Juniperus thurifera. New Phytol. 182:687-697.

Sklensky, D.E. and P.J. Davies. 2011. Resource partitioning to male and female flowers of Spinacia oleracea L. in relation to whole-plant monocarpic senescence. J. Expt. Bot. 62:4323-4336.

Spundova, M., H. Popelkova, P. Llik, J. Skotnica, R. Novotny, and J. Naus. 2003. Ultra-structural and functional changes in the chloroplasts of detached barley leaves senescing under dark and light conditions. J. Plant Physiol. 160:1051-1058.

Stehlik, I., J. Friedman, and S.C.H. Barrett. 2008. Environmental influence on primary sex ratio in a dioecious plant. Proc. Natl. Acad. Sci. USA 105:10847-10852.

Subhan, D. and S.D.S. Murthy. 2001. Senescence retarding effect of metal ions: Pigment and protein contents and photochemical activities of detached primary leaves of wheat. Photosynthetica 39:53-58.

Tanaka, K., Y. Suda, N. Kondo, and K. Sugahara. 1985. O $\mathrm{O}_{3}$ tolerance and the ascorbate-dependent $\mathrm{H}_{2} \mathrm{O}_{2}$ decomposing system in chloroplasts. Plant Cell Physiol. 26:1425-1431.

Vallejos, R.H., J.L. Arana, and R.A. Ravizzini. 1983. Changes in activity and structure of the chloroplast proton ATPase induced by illumination of spinach leaves. J. Biol. Chem. 258:7317-7321.

Veljovic-Jovanovic, S., B. Kukavica, B. Stevanovic, and F. Navari-Izzo. 2006. Senescence- and drought-related changes in peroxidase and superoxide dismutase isoforms in leaves of Ramonda serbica. J. Expt. Bot. 57:1759-1768.

Veljovic-Jovanovic, S., G. Noctor, and C.H. Foyer. 2002. Are leaf hydrogen peroxide concentrations commonly overestimated? The potential influence of artefactual interference by tissue phenolics and ascorbate. Plant Physiol. Biochem. 40:501-507.

Whitehead, D., M.M. Barbour, K.L. Griffin, M.H. Turnbull, and D.T. Tissue. 2011. Effects of leaf age and tree size on stomatal and mesophyll limitations to photosynthesis in mountain beech 
(Nothofagus solandrii var. cliffortiodes). Tree Physiol. 31:985996.

Zhang, C.J., G.X. Chen, X.X. Gao, and C.J. Chu. 2006. Photosynthetic decline in flag leaves of two field-grown spring wheat cultivars with different senescence properties. S. Afr. J. Bot. 72:15-23.

Zhang, C.J., H.J. Chu, G.X. Chen, D.W. Shi, M. Zuo, J. Wang, C.G. Lu, P. Wang, and L. Chen. 2007. Photosynthetic and biochemical activities in flag leaves of a newly developed superhigh-yield hybrid rice (Oryza sativa) and its parents during the reproductive stage. J. Plant Res. 120:209-217.

Zhang, M.P., C.J. Zhang, G.H. Yu, Y.Z. Jiang, R.J. Strasser, Z.Y. Yuan, X.S. Yang, and G.X. Chen. 2010a. Changes in chloroplast ultrastructure, fatty acid components of thylakoid membrane and chlorophyll a fluorescence transient in flag leaves of a super-highyield hybrid rice and its parents during the reproductive stage. J. Plant Physiol. 167:277-285.

Zhang, S., S. Lu, X. Xu, H. Korpelainen, and C.Y. Li. 2010b. Changes in antioxidant enzyme activities and isozyme profiles in leaves of male and female Populus cathayana infected with Melampsora laricipopulina. Tree Physiol. 30:116-128.

Zhang, S., H. Jiang, S. Peng, H. Korpelainen, and C.Y. Li. 2011. Sexrelated differences in morphological, physiological, and ultrastructural responses of Populus cathayana to chilling. J. Expt. Bot. 62:675-686.

Zhao, H.X., Y. Li, L.B. Duan, H. Korpelainen, and C.Y. Li. 2009. Sexrelated adaptive responses of Populus cathayana to photoperiod transitions. Plant Cell Environ. 32:1401-1411.

Zhao, S.J. and D.Q. Li. 1999. The modern experimental directory on plant physiology. Science Press, Beijing, China.

Zhu, X.Y., G.C. Chen, and C.L. Zhang. 2001. Photosynthetic electron transport, photophosphorylation, and antioxidants in two ecotypes of reed (Phragmites Communis Trin.) from different habitats. Photosynthetica 39:183-189.

Zimmermann, P. and U. Zentgraf. 2005. The correlation between oxidative stress and leaf senescence during plant development. Cell. Mol. Biol. Lett. 10:515-534. 